\title{
Bianchi type I cyclic cosmology from Lie-algebraically deformed phase space
}

\author{
Babak Vakili ${ }^{1 *}$ and Nima Khosravi ${ }^{2 \dagger}$ \\ ${ }^{1}$ Department of Physics, Azad University of Chalous, P. O. Box 46615-397, Chalous, Iran \\ ${ }^{2}$ Department of Physics, Shahid Beheshti University, G. C., Evin, Tehran 19839, Iran
}

\begin{abstract}
We study the effects of noncommutativity, in the form of a Lie-algebraically deformed Poisson commutation relations, on the evolution of a Bianchi type I cosmological model with a positive cosmological constant. The phase space variables turn out to correspond to the scale factors of this model in $x, y$ and $z$ directions. According to the conditions that the structure constants (deformation parameters) should satisfy, we argue that there are two types of noncommutative phase space with Lie-algebraic structure. The exact classical solutions in commutative and type I noncommutative cases are presented. In the framework of this type of deformed phase space, we investigate the possibility of building a Bianchi I model with cyclic scale factors in which the size of the Universe in each direction experiences an endless sequence of contractions and reexpansions. We also obtain some approximate solutions for the type II noncommutative structure by numerical methods and show that the cyclic behavior is repeated as well. These results are compared with the standard commutative case, and similarities and differences of these solutions are discussed.
\end{abstract}

PACS numbers: $98.80 .-\mathrm{k}, 04.60 . \mathrm{Kz}, 11.10 \mathrm{Nx}$

Keywords: Cyclic cosmology, Lie-algebraic deformation, Bianchi type I model

\section{Introduction}

Since 1947 when noncommutativity between space-time coordinates was first introduced by Snyder [1], many efforts have been made in this area and the corresponding results have been followed by a number of works, the main motivations of which lie in the results of string theory [2]. Although the interest in noncommutative theories has been gathering pace in recent years because of strong motivations in the development of string and M-theories, they may also be justified in their own right because of the interesting predictions they have made in particle physics [3], quantum [4] and classical mechanics [5]. In a general classification there are three sorts of noncommutative space-time with the following deformed algebra between coordinates [6]

- Deformed Poisson algebra with canonical structure

$$
\left\{x_{\mu}, x_{\nu}\right\}=\theta_{\mu \nu}
$$

where the tensor (with constant components) $\theta_{\mu \nu}$ is assumed to be antisymmetric.

- Deformed Poisson algebra with Lie-algebraic structure

$$
\left\{x_{\mu}, x_{\nu}\right\}=\theta_{\mu \nu}^{\lambda} x_{\lambda}
$$

*email: b-vakili@iauc.ac.ir

†email: n-khosravi@sbu.ac.ir 
where the (constant) deformation parameters $\theta_{\mu \nu}^{\lambda}$ are assumed to be antisymmetric to lower indices.

- Deformed Poisson algebra with quantum space structure

$$
\left\{x_{\mu}, x_{\nu}\right\}=\theta_{\mu \nu}^{\alpha \beta} x_{\alpha} x_{\beta},
$$

where again the (constant) deformation parameters $\theta_{\mu \nu}^{\alpha \beta}$ are assumed to be antisymmetric to lower indices.

In general, as is clear from (1)-(3), noncommutativity in their original form imply a noncommutative underlying geometry for space-time. However, formulation of gravity in a noncommutative space-time is highly nonlinear, rendering the setting up of cosmological models difficult. In this respect, a different approach to noncommutativity is through its introduction in the phase space constructed by minisuperspace fields and their conjugate momenta. Alternatively, in cosmological systems, since the scale factors, matter fields and their conjugate momenta play the role of dynamical variables of the system, introducing noncommutativity in the corresponding phase space is particularly relevant [7]. This means that in the relations (1)-(3) the space-time coordinates can be replaced by the phase space coordinates to construct a noncommutative or deformed phase space. In this sense, one can introduce different kinds of noncommutativity between different dynamical variables of the corresponding phase space and the main aim is to study the aspects related to the application of noncommutativity in the framework of minisuperspace reduction of dynamics.

In this paper we are going to investigate the impact of the deformation of Lie-algebraic type on the cosmological dynamics of Bianchi type I model. Since the Bianchi models have different scale factors in different directions, they are suitable candidates for studying noncommutative cosmology. Here, our aim is to introduce Lie-algebraic noncommutative scale factors in Bianchi type I space-time and compare and contrast their solutions to that of the conventional case. It should be emphasized that when we speak of noncommutativity in this work, we mean noncommutativity in the fields (scale factors) and not the coordinates, that is to say that we study noncommutativity within the context of phase space only. We shall see that the main feature of the resulting noncommutative model is constructing a scenario of cyclic cosmology governed by introducting a Lie-algebraic deformation in the phase space of Bianchi type I space-time. In such a scenario the history of the Universe (in each direction) is periodic, i.e., the Universe undergoes a periodic sequence of expansion and contraction phases. The existence of cyclic solutions in the FRW models was first introduced by Tolman in [8] and then the early results in this subject have been followed by a number of works, see [9] and for a review see [10]. In these works cyclic cosmologies which are an extension of bouncing scenarios, are used as an alternative for conventional inflationary cosmology. Instead of a continuously expansion after big-bang, the bang is replaced by a transition to an earlier phase of evolution.

The structure of the paper is as follows: after a brief review of Bianchi type I cosmology with a cosmological constant in section 2 , we deal with a Lie-algebraically deformed phase space in section 3. In this section by imposing some constraints (Jacobi identity) on the structure constants of the Lie algebra, we present two kinds of Lie-algebraic deformed of Poisson brackets according to which we write the (Poisson) commutation relations between the dynamical variables of the Bianchi I model. The exact solutions in type I noncommutative cases are presented. We also obtain, in this section, some approximate solutions for the type II noncommutative structure by numerical methods. Finally, in section 4 we summarize and discuss the results.

\section{Bianchi type I cosmology with cosmological constant}

In this section we make a quick review of some of the important results in the Bianchi type I model and obtain its Lagrangian and Hamiltonian in the ADM decomposition, for more details see [11]. The simplest generalization of the flat FRW cosmology is the Bianchi type I Universe which has the metric

$$
d s^{2}=-N^{2}(t) d t^{2}+a^{2}(t) d x^{2}+b^{2}(t) d y^{2}+c^{2}(t) d z^{2} .
$$


In this metric $N(t)$ is the lapse function and there are three functions $a(t), b(t)$ and $c(t)$, to be determined by the Einstein field equations, and are the scale factors of the corresponding Universe in the $x, y$ and $z$ directions respectively. The scale factors in different directions are allowed to vary independently of each other. This metric is the simplest anisotropic and homogeneous cosmological model which, upon making the scale factors equal, becomes the flat FRW metric. In general, the nine Bianchi (class A) models are the most general homogeneous cosmological solutions of the Einstein field equations which admit a three-dimensional isometry group, i.e. their spatially homogeneous sections are invariant under the action of a three-dimensional Lie group. To transform the Lagrangian of the dynamical system which corresponds to the Bianchi cosmologies to a more manageable form, it is useful to introduce the following change of variables

$$
a(t)=e^{u(t)+v(t)+\sqrt{3} w(t)}, \quad b(t)=e^{u(t)+v(t)-\sqrt{3} w(t)}, \quad c(t)=e^{u(t)-2 v(t)} .
$$

In the Misner notation [12], the metric of the Bianchi models can be written in terms of these new variables as

$$
d s^{2}=-N^{2}(t) d t^{2}+e^{2 u(t)} e^{2 \beta_{i j}(t)} \omega^{i} \otimes \omega^{j},
$$

where $V(t)=e^{3 u(t)}=a b c$ is the comoving volume of the Universe and $\beta_{i j}$ determines the anisotropic parameters $v(t)$ and $w(t)$ as follows

$$
\beta_{i j}=\operatorname{diag}(v+\sqrt{3} w, v-\sqrt{3} w,-2 v) .
$$

Also, in metric (6), the one-forms $\omega^{i}$ represent the invariant one-forms of the corresponding isometry group and satisfy the following Lie algebra

$$
d \omega^{i}=-\frac{1}{2} C_{j k}^{i} \omega^{j} \wedge \omega^{k},
$$

where $C_{j k}^{i}$ are the structure constants. Indeed, the Bianchi models are grouped by their structure constants into classes A and B. Because of the difficultly in formulating the class B Bianchi models in the context of the ADM decomposition and canonical quantization [13], it is usually the case that one confines attention to the class A models where the structure constants obey the relation $C_{j i}^{i}=0$.

The Einstein-Hilbert action is given by (we work in units where $c=\hbar=16 \pi G=1$ )

$$
\mathcal{S}=\int d^{4} x \sqrt{-g}(\mathcal{R}-\Lambda)
$$

where $g$ is the determinant of the metric, $\mathcal{R}$ is the scalar curvature of the space-time metric (6) and $\Lambda$ is the cosmological constant. In terms of the ADM variables, action (9) can be written as [14]

$$
\mathcal{S}=\int d t d^{3} x \mathcal{L}=\int d t d^{3} x N \sqrt{h}\left(K_{i j} K^{i j}-K^{2}+R-\Lambda\right),
$$

where $K_{i j}$ are the components of extrinsic curvature (second fundamental form) which represent how much the spatial space $h_{i j}$ is curved in the way it sits in the space-time manifold. Also, $h$ and $R$ are the determinant and scalar curvature of the spatial geometry $h_{i j}$ respectively, and $K$ represents the trace of $K_{i j}$. The extrinsic curvature is given by

$$
K_{i j}=\frac{1}{2 N}\left(N_{i \mid j}+N_{j \mid i}-\frac{\partial h_{i j}}{\partial t}\right),
$$

where $N_{i \mid j}$ represents the covariant derivative with respect to $h_{i j}$. Using (6) and (7) we obtain the nonvanishing components of the extrinsic curvature and its trace as follows

$$
\left\{\begin{array}{l}
K_{11}=-\frac{1}{N}(\dot{u}+\dot{v}+\sqrt{3} \dot{w}) e^{2(u+v+\sqrt{3} w)} \\
K_{22}=-\frac{1}{N}(\dot{u}+\dot{v}-\sqrt{3} \dot{w}) e^{2(u+v-\sqrt{3} w)} \\
K_{33}=-\frac{1}{N}(\dot{u}-2 \dot{v}) e^{2(u-2 v)} \\
K=-3 \frac{\dot{u}}{N}
\end{array}\right.
$$


where a dot represents differentiation with respect to $t$. The scalar curvature $R$ of a spatial hypersurface is a function of $v$ and $w$ and can be write in terms of the structure constants as [15]

$$
R=C_{j k}^{i} C_{m n}^{l} h_{i l} h^{k m} h^{j n}+2 C_{j k}^{i} C_{l i}^{k} h^{j l} .
$$

The Lagrangian for the Bianchi class A models may now be written by substituting the above results into action (10), giving

$$
\mathcal{L}=\frac{6 e^{3 u}}{N}\left(-\dot{u}^{2}+\dot{v}^{2}+\dot{w}^{2}\right)+N e^{3 u}(R-\Lambda) .
$$

The momenta conjugate to the dynamical variables are given by

$$
p_{u}=\frac{\partial \mathcal{L}}{\partial \dot{u}}=-\frac{12}{N} \dot{u} e^{3 u}, \quad p_{v}=\frac{\partial \mathcal{L}}{\partial \dot{v}}=\frac{12}{N} \dot{v} e^{3 u}, \quad p_{w}=\frac{\partial \mathcal{L}}{\partial \dot{w}}=\frac{12}{N} \dot{w} e^{3 u},
$$

leading to the following Hamiltonian

$$
\mathcal{H}=\frac{1}{24} N e^{-3 u}\left(-p_{u}^{2}+p_{v}^{2}+p_{w}^{2}\right)-N e^{3 u}(R-\Lambda) .
$$

The preliminary set-up for writing the dynamical equations is now complete. The cosmological dynamics of the Bianchi models are studied in many works [11]-[16]. In the following, we shall consider only the simplest Bianchi class A model, namely type I. The structure constants of the Bianchi type I are all zero, that is $C_{j k}^{i}=0$. It then follows from equation (13) that $R=0$. Thus, with the choice of the cosmic time gauge $N=1$, the Hamiltonian can be written as

$$
\mathcal{H}=\frac{1}{24} e^{-3 u}\left(-p_{u}^{2}+p_{v}^{2}+p_{w}^{2}\right)+\Lambda e^{3 u} .
$$

The Poisson brackets for the phase space variables are

$$
\left\{x_{i}, x_{j}\right\}=0, \quad\left\{p_{i}, p_{j}\right\}=0, \quad\left\{x_{i}, p_{j}\right\}=\delta_{i j}
$$

where $x_{i}(i=1,2,3)=v, w, u$ and $p_{i}(i=1,2,3)=p_{v}, p_{w}, p_{u}$. Therefore, the classical dynamics is governed by the Hamiltonian equations, that is

$$
\left\{\begin{array}{l}
\dot{u}=\{u, \mathcal{H}\}=-\frac{1}{12} e^{-3 u} p_{u}, \quad \dot{p_{u}}=\left\{p_{u}, \mathcal{H}\right\}=\frac{1}{8} e^{-3 u}\left(-p_{u}^{2}+p_{v}^{2}+p_{w}^{2}\right)-3 \Lambda e^{3 u}, \\
\dot{v}=\{v, \mathcal{H}\}=\frac{1}{12} e^{-3 u} p_{v}, \quad \dot{p_{v}}=\left\{p_{v}, \mathcal{H}\right\}=0 \\
\dot{w}=\{w, \mathcal{H}\}=\frac{1}{12} e^{-3 u} p_{w}, \quad \dot{p_{w}}=\left\{p_{w}, \mathcal{H}\right\}=0 .
\end{array}\right.
$$

We also have the constraint equation $\mathcal{H}=0$, from which we obtain

$$
e^{-3 u}\left(-p_{u}^{2}+p_{v}^{2}+p_{w}^{2}\right)=-24 \Lambda e^{3 u}
$$

Using this relation in eliminating $p_{u}$ from the first equations of the system (19) results

$$
\ddot{u}+3 \dot{u}^{2}=\frac{\omega^{2}}{3},
$$

where $\omega=\sqrt{\frac{3 \Lambda}{2}}$. The solution of this equation can be read as

$$
u(t)=\frac{1}{3} C+\frac{1}{3} \ln [\sinh \omega(t-\delta)],
$$

where $C$ and $\delta$ are integrating constants. Since $\delta$ only corresponds to a shift in the big-bang singularity, we can put it equal to zero. The last two equations of the system (19) indicate that $v(t)$ and $w(t)$ 
obey the equations of motion $\dot{v}=\frac{1}{12} p_{0 v} e^{-3 u}$ and $\dot{w}=\frac{1}{12} p_{0 w} e^{-3 u}$, where we take $p_{v}=p_{0 v}=$ cons. and $p_{w}=p_{0 w}=$ cons. Upon substituting the relation (22) into these equations, they can easily be integrated to yield

$$
v(t)=\frac{p_{0 v}}{12 e^{C} \omega} \ln \left[\tanh \frac{\omega t}{2}\right], \quad w(t)=\frac{p_{0 w}}{12 e^{C} \omega} \ln \left[\tanh \frac{\omega t}{2}\right] .
$$

Now, these solutions must satisfy the zero energy condition, $\mathcal{H}=0$. Thus, substitution of equations (22) and (23) into (20) gives a relation between integration constants as

$$
C=\ln \frac{\sqrt{p_{0 v}^{2}+p_{0 w}^{2}}}{4 \omega} .
$$

From these relations one can calculate all of the physical quantities of observational interest in cosmology. In analogy with a FRW Universe, we can define a Hubble parameter corresponding to each of the three different directions

$$
H_{a}=\frac{\dot{a}}{a}, \quad H_{b}=\frac{\dot{b}}{b}, \quad H_{c}=\frac{\dot{c}}{c}
$$

in terms of which the average Hubble parameter is defined as

$$
H=\frac{1}{3}\left(H_{a}+H_{b}+H_{c}\right)=\dot{u} .
$$

The directional Hubble parameter $H_{i}$ measures the expansion rate of the Universe in the direction $x_{i}$ while the average Hubble parameter $H$ measures its volumetric expansion rate. We will also define the expansion scalar $\Theta=3 H$, the shear scalar $\sigma^{2}=\frac{1}{2}\left(\sum_{i=1}^{3} H_{i}^{2}-3 H^{2}\right)=3\left(\dot{v}^{2}+\dot{w}^{2}\right)$, which measures the degree of anisotropy of the space-time and the average deceleration parameter $q=\frac{d}{d t}\left(\frac{1}{H}\right)-1$, where as is well known is indicated by how much the expansion of the Universe is slowing down. If the expansion is speeding up, for which there appears to be some recent evidence, then this parameter will be negative. Now, let us return to the variables $a_{i}(t)\left[a_{1}(t)=a(t), a_{2}(t)=b(t)\right.$ and $\left.a_{3}(t)=c(t)\right]$ using the transformation (5), in terms of which we obtain the corresponding scale factors for the Bianchi I cosmology as

$$
a_{i}(t)=\left(\frac{\sqrt{p_{0 v}^{2}+p_{0 w}^{2}}}{2 \omega}\right)^{1 / 3} \sinh ^{\frac{1}{3}+s_{i}} \frac{\omega t}{2} \cosh ^{\frac{1}{3}-s_{i}} \frac{\omega t}{2},
$$

where the constants $s_{i}$ are defined as

$$
s_{1}=\frac{p_{0 v}+\sqrt{3} p_{0 w}}{3 \sqrt{p_{0 v}^{2}+p_{0 w}^{2}}}, \quad s_{2}=\frac{p_{0 v}-\sqrt{3} p_{0 w}}{3 \sqrt{p_{0 v}^{2}+p_{0 w}^{2}}}, \quad s_{3}=\frac{-2 p_{0 v}}{3 \sqrt{p_{0 v}^{2}+p_{0 w}^{2}}},
$$

and satisfy the following relations

$$
s_{1}+s_{2}+s_{3}=0, \quad s_{1}^{2}+s_{2}^{2}+s_{3}^{2}=\frac{2}{3} .
$$

From these equations we obtain, the directional Hubble parameters

$$
H_{i}=\frac{\dot{a}_{i}}{a_{i}}=\frac{s_{i} \omega}{\sinh \omega t}+\frac{\omega}{3} \operatorname{coth} \omega t,
$$

the comoving volume

$$
V(t)=\frac{\sqrt{p_{0 v}^{2}+p_{0 w}^{2}}}{4 \omega} \sinh \omega t
$$



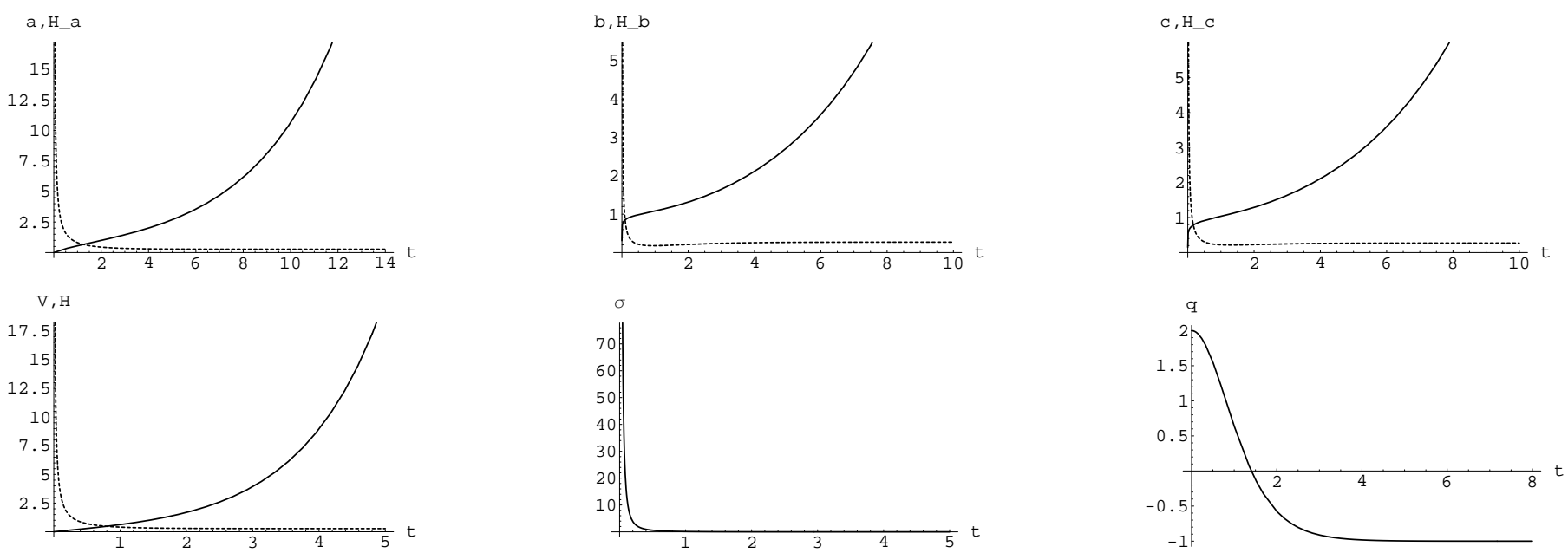

Figure 1: Top: Behavior of the scale factors $a(t), b(t)$ and $c(t)$ (solid lines) and their corresponding Hubble parameters (dashed lines) as a function of time. Bottom: The comoving volume $V(t)=e^{3 u}=a b c$ (solid line) and its corresponding Hubble parameter (dashed line), the shear scalar and the deceleration parameter. The figures are plotted for numerical values $\Lambda=1, p_{0 v}=1$ and $p_{0 w}=2$.

the average Hubble parameter

$$
H(t)=\frac{\omega}{3} \operatorname{coth} \omega t
$$

the shear scalar

$$
\sigma(t)=\frac{\sqrt{3}}{4} \frac{1}{\sinh \omega t}
$$

and the deceleration parameter

$$
q(t)=\frac{3}{\cosh ^{2} \omega t}-1
$$

In figure 1, we have shown the behavior of the scale factors, Hubble parameters, comoving volume, shear scalar and deceleration parameter for typical values of the parameters. It is seen that the evolution of the Universe begins with a big-bang singularity at $t=0$ with a high degree of anisotropy and then follows an expansion phase in all directions. Since we have negative acceleration (positive deceleration parameter) at early times, the Universe decelerates its volumetric expansion in this era. On the other hand, the positive acceleration (negative deceleration parameter) is occurred for late times which means that the Universe currently accelerates its volumetric expansion. While the volume of the Universe and its scale factors in different directions increase monotonically, the shear scalar decreases monotonically and tends to zero as $t \rightarrow \infty$. This means at late time the model approaches that of the flat FRW Universe with cosmological constant, i.e., the Universe eventually evolves to a phase where it is close to a de Sitter solution.

\section{Lie-algebraically deformed phase space}

We now focus attention on the study of the effects of Lie-algebraic noncommutativity on the cosmological dynamic of the Bianchi type I model described above. This kind of noncommutativity is described by a deformed Poisson algebra between the phase space variables with the Lie-algebraic structure as follows

$$
\left\{x_{i}, x_{j}\right\}=\theta_{i j}^{k} x_{k},
$$

where the constants $\theta_{i j}^{k}$ are assumed to be antisymmetric with respect to their lower indices. To guarantee the Jacobi identity

$$
\left\{\left\{x_{i}, x_{j}\right\}, x_{k}\right\}+\left\{\left\{x_{j}, x_{k}\right\}, x_{i}\right\}+\left\{\left\{x_{k}, x_{i}\right\}, x_{j}\right\}=0,
$$


these parameters are also subject to the following conditions

$$
\theta_{i j}^{l} \theta_{l k}^{m}+\theta_{k i}^{l} \theta_{l j}^{m}+\theta_{j k}^{l} \theta_{l i}^{m}=0
$$

Following [17], we consider the Poisson brackets between the dynamical variables and their momenta as

$$
\left\{x_{i}, p_{j}\right\}=\delta_{i j}+\bar{\theta}_{i j}^{k} x_{k}+\tilde{\theta}_{i j}^{k} p_{k}, \quad\left\{p_{i}, p_{j}\right\}=0,
$$

in which the new noncommutative parameters $\bar{\theta}$ and $\tilde{\theta}$ with vanishing diagonal elements are introduced and furthermore, we assume that the momenta (Poisson) commute with each other. For our model at hand, $x_{i}(i=1,2,3)=v, w, u$ and $p_{i}(i=1,2,3)=p_{v}, p_{w}, p_{u}$, as before. In the phase space with coordinates $\vec{\eta}=(\mathbf{x}, \mathbf{p})$, in addition of the identity (36), the following Jacobi identities should also hold

$$
\begin{aligned}
& \left\{\left\{x_{i}, x_{j}\right\}, p_{k}\right\}+\left\{\left\{x_{j}, p_{k}\right\}, x_{i}\right\}+\left\{\left\{p_{k}, x_{i}\right\}, x_{j}\right\}=0, \\
& \left\{\left\{x_{i}, p_{j}\right\}, p_{k}\right\}+\left\{\left\{p_{j}, p_{k}\right\}, x_{i}\right\}+\left\{\left\{p_{k}, x_{i}\right\}, p_{j}\right\}=0 .
\end{aligned}
$$

These relations impose some more constraints on the noncommutative parameters as

$$
\left\{\begin{array}{l}
\theta_{i j}^{k}+\tilde{\theta}_{i k}^{j}-\tilde{\theta}_{j k}^{i}=0, \\
\theta_{i j}^{l} \bar{\theta}_{l k}^{m}+\bar{\theta}_{j k}^{l} \theta_{l i}^{m}-\bar{\theta}_{i l}^{m} \tilde{\theta}_{j k}^{l}-\bar{\theta}_{i k}^{l} \theta_{l j}^{m}+\tilde{\theta}_{i k}^{l} \bar{\theta}_{j l}^{m}=0, \\
\theta_{i j}^{l} \tilde{\theta}_{l k}^{m}+\tilde{\theta}_{i k}^{l} \tilde{\theta}_{j l}^{m}-\tilde{\theta}_{j k}^{l} \tilde{\theta}_{i l}^{m}=0, \\
\bar{\theta}_{i j}^{k}-\bar{\theta}_{i k}^{j}=0, \\
\bar{\theta}_{i j}^{l} \bar{\theta}_{l k}^{m}-\bar{\theta}_{i k}^{l} \bar{\theta}_{l j}^{m}=0, \\
\bar{\theta}_{i j}^{l} \tilde{\theta}_{l k}^{m}-\bar{\theta}_{i k}^{l} \tilde{\theta}_{l j}^{m}=0 .
\end{array}\right.
$$

Now, the equations (37) and (41) should be solved to determine the noncommutative parameters. Here we will not deal with the details of the methods through which one can find these solutions and only refer to the Refs. [17] where the following two kinds of solutions to the equations (37) and (41) are proposed.

- Type I: For this type of solution, the nonvanishing noncommutative parameters are as follows (the antisymmetric counterparts of $\theta_{i j}^{k}$ should be also considered)

$$
\theta_{13}^{2}=-\theta_{23}^{1}=\theta, \quad \tilde{\theta}_{32}^{1}=-\tilde{\theta}_{31}^{2}=\theta .
$$

In this case the Poisson brackets of the phase space variables correspond to our cosmological setting read as

$$
\left\{\begin{array}{l}
\{v, w\}=0, \quad\{w, u\}=-\theta v, \quad\{u, v\}=-\theta w \\
\left\{u, p_{u}\right\}=1, \quad\left\{u, p_{v}\right\}=-\theta p_{w}, \quad\left\{u, p_{w}\right\}=\theta p_{v} \\
\left\{v, p_{u}\right\}=0, \quad\left\{v, p_{v}\right\}=1, \quad\left\{v, p_{w}\right\}=0, \\
\left\{w, p_{u}\right\}=0, \quad\left\{w, p_{v}\right\}=0, \quad\left\{w, p_{w}\right\}=1
\end{array}\right.
$$

- Type II: For this type of solution, we have the following nonvanishing noncommutative parameters (and also the antisymmetric counterparts of $\theta_{i j}^{k}$ )

$$
\theta_{13}^{2}=-\theta_{23}^{1}=\theta, \quad \tilde{\theta}_{32}^{1}=-\tilde{\theta}_{31}^{2}=\theta, \quad \bar{\theta}_{31}^{2}=\bar{\theta}_{32}^{1}=-\bar{\theta} .
$$


In this case the Poisson brackets of the phase space variables correspond to our cosmological setting read as

$$
\left\{\begin{array}{l}
\{v, w\}=0, \quad\{w, u\}=-\theta v, \quad\{u, v\}=-\theta w \\
\left\{u, p_{u}\right\}=1, \quad\left\{u, p_{v}\right\}=-\bar{\theta} w-\theta p_{w}, \quad\left\{u, p_{w}\right\}=-\bar{\theta} v+\theta p_{v} \\
\left\{v, p_{u}\right\}=0, \quad\left\{v, p_{v}\right\}=1, \quad\left\{v, p_{w}\right\}=0, \\
\left\{w, p_{u}\right\}=0, \quad\left\{w, p_{v}\right\}=0, \quad\left\{w, p_{w}\right\}=1
\end{array}\right.
$$

The Poisson bracket for any phase space function can be straightforward and obtained from (43) and (45) and reads

$$
\{f(\mathbf{x}, \mathbf{p}), g(\mathbf{x}, \mathbf{p})\}=\left\{\eta^{A}, \eta^{B}\right\} \frac{\partial f}{\partial \eta^{A}} \frac{\partial g}{\partial \eta^{B}},
$$

where $\vec{\eta}=(\mathbf{x}, \mathbf{p})=\left(v, w, u, p_{v}, p_{w}, p_{u}\right)$. The time evolution of such a function is thus given by

$$
\frac{d f}{d t}=\{f, \mathcal{H}\} .
$$

For deformed phase space of type I the cosmological dynamics of the Bianchi-I model are

$$
\left\{\begin{array}{l}
\dot{u}=\{u, \mathcal{H}\}=-\frac{1}{12} e^{-3 u} p_{u} \\
\dot{v}=\{v, \mathcal{H}\}=\theta w\left[-\frac{1}{8} e^{-3 u}\left(-p_{u}^{2}+p_{v}^{2}+p_{w}^{2}\right)+3 \Lambda e^{3 u}\right]+\frac{1}{12} e^{-3 u} p_{v}, \\
\dot{w}=\{w, \mathcal{H}\}=-\theta v\left[-\frac{1}{8} e^{-3 u}\left(-p_{u}^{2}+p_{v}^{2}+p_{w}^{2}\right)+3 \Lambda e^{3 u}\right]+\frac{1}{12} e^{-3 u} p_{w} \\
\dot{p_{u}}=\left\{p_{u}, \mathcal{H}\right\}=-3 \Lambda e^{3 u}+\frac{1}{8} e^{-3 u}\left(-p_{u}^{2}+p_{v}^{2}+p_{w}^{2}\right) \\
\dot{p_{v}}=\left\{p_{v}, \mathcal{H}\right\}=\theta p_{w}\left[-\frac{1}{8} e^{-3 u}\left(-p_{u}^{2}+p_{v}^{2}+p_{w}^{2}\right)+3 \Lambda e^{3 u}\right] \\
\dot{p_{w}}=\left\{p_{w}, \mathcal{H}\right\}=-\theta p_{v}\left[-\frac{1}{8} e^{-3 u}\left(-p_{u}^{2}+p_{v}^{2}+p_{w}^{2}\right)+3 \Lambda e^{3 u}\right]
\end{array}\right.
$$

As before, assuming the full Einstein field equations hold, this implies that the corresponding Hamiltonian must vanish, that is $\mathcal{H}=0$, which yields

$$
\frac{1}{8} e^{-3 u}\left(-p_{u}^{2}+p_{v}^{2}+p_{w}^{2}\right)=-3 \Lambda e^{3 u}
$$

We see that the noncommutativity does not affect the dynamics of $u$ and $p_{u}$. Therefore, we have

$$
u(t)=\frac{1}{3} C+\frac{1}{3} \ln [\sinh \omega t], \quad p_{u}(t)=-4 \omega e^{C} \cosh \omega t,
$$

which means that the volumetric expansion of the Universe given by $V(t)=e^{3 u(t)}$ is the same as the ordinary Bianchi I model described in the previous section. Substituting these results into the rest of the equations of the system (48), we are led to

$$
\left\{\begin{array}{l}
\dot{v}=6 \Lambda \theta e^{C} w \sinh \omega t+\frac{1}{12 e^{C} \sinh \omega t} p_{v} \\
\dot{w}=-6 \Lambda \theta e^{C} v \sinh \omega t+\frac{1}{12 e^{C} \sinh \omega t} p_{w} \\
\dot{p_{v}}=6 \Lambda \theta e^{C} p_{w} \sinh \omega t \\
\dot{p_{w}}=-6 \Lambda \theta e^{C} p_{v} \sinh \omega t
\end{array}\right.
$$


From the third and fourth equations of this system, we obtain

$$
\ddot{p_{v}}-\omega \dot{p_{v}} \operatorname{coth} \omega t+36 \Lambda^{2} \theta^{2} e^{2 C} p_{v} \sinh ^{2} \omega t=0,
$$

where after integration we get the following expressions for $p_{v}$ and $p_{w}$

$$
\begin{aligned}
& p_{v}(t)=p_{0 v} \cos \left(\frac{6 \Lambda \theta e^{C}}{\omega} \cosh \omega t\right)+p_{0 w} \sin \left(\frac{6 \Lambda \theta e^{C}}{\omega} \cosh \omega t\right), \\
& p_{w}(t)=-p_{0 v} \sin \left(\frac{6 \Lambda \theta e^{C}}{\omega} \cosh \omega t\right)+p_{0 w} \cos \left(\frac{6 \Lambda \theta e^{C}}{\omega} \cosh \omega t\right),
\end{aligned}
$$

in which we have set the integration constants as $p_{0 v}$ and $p_{0 w}$ to achieve the correct results in the limit $\theta \rightarrow 0$. With these results at hand we can integrate the two first equations of the system (51) to obtain

$$
\begin{aligned}
v(t)= & C_{1} \cos \left(\frac{6 \Lambda \theta e^{C}}{\omega} \cosh \omega t\right)+C_{2} \sin \left(\frac{6 \Lambda \theta e^{C}}{\omega} \cosh \omega t\right)+ \\
& \frac{1}{12 e^{C} \omega} \ln \left(\tanh \frac{\omega t}{2}\right)\left\{p_{0 v} \cos \left(\frac{6 \Lambda \theta e^{C}}{\omega} \cosh \omega t\right)+p_{0 w} \sin \left(\frac{6 \Lambda \theta e^{C}}{\omega} \cosh \omega t\right)\right\}, \\
w(t)= & C_{2} \cos \left(\frac{6 \Lambda \theta e^{C}}{\omega} \cosh \omega t\right)-C_{1} \sin \left(\frac{6 \Lambda \theta e^{C}}{\omega} \cosh \omega t\right)+ \\
& \frac{1}{12 e^{C} \omega} \ln \left(\tanh \frac{\omega t}{2}\right)\left\{p_{0 w} \cos \left(\frac{6 \Lambda \theta e^{C}}{\omega} \cosh \omega t\right)-p_{0 v} \sin \left(\frac{6 \Lambda \theta e^{C}}{\omega} \cosh \omega t\right)\right\},
\end{aligned}
$$

where $C_{1}$ and $C_{2}$ are integration constants. The requirement that the deformed Hamiltonian constraints (49) should hold during the evolution of the system leads again to the relation (24) between integrating constants. In figure 2 we have plotted the cosmological functions corresponding to the above solutions. As is clear from this figure the volumetric expansion, average Hubble and deceleration parameters of the deformed model are exactly the same as the usual Bianchi I model. This is because these parameters come from the function $u(t)$ which is not affected by the deformation on the phase space. However, while like the usual model, the volume of the Universe increases continuously, the evolution of the scale factors in three directions is quite different in comparison with the usual Bianchi I model. We see that the scale factors behave cyclically, i.e., they undergo a periodic sequence of contractions and expansions phases. In contrast to the conventional big-bang inflationary cosmology described in the previous section in which the Universe begins with the big-bang singularity and expands forever, here we have an alternative in which the bang is replaced by a transition to an earlier phase of evolution. In each cycle of such a cosmology the scale factors begin their evolution with a big-bang and end in a big-crunch, only to emerge in a big-bang once again. Cyclic cosmologies have been extensively studied in literature in an attempt to solve some problems of the standard cosmological model such as the flatness problem, the horizon problem, the coincidence problem, the problem of initial conditions etc. [10]. In general, a cyclic Universe can be generated by adding a matter or a field that will produce a bounce, and then explore what conditions are to be imposed on it to produce oscillations. In the above analysis we provided an alternative method to construct a Bianchi type I cosmology with cyclic scale factors by introducing a deformation of Lie-algebraic type on the phase space of this model. In our model although the expansion of the whole volume of the Universe is speeding up, for which there appears to be some recent evidence, from the evolution of the scale factors point of view, the history of the Universe is periodic and all of the key events that construct the large scale structure of the observable Universe occurred a cycle ago. An important 

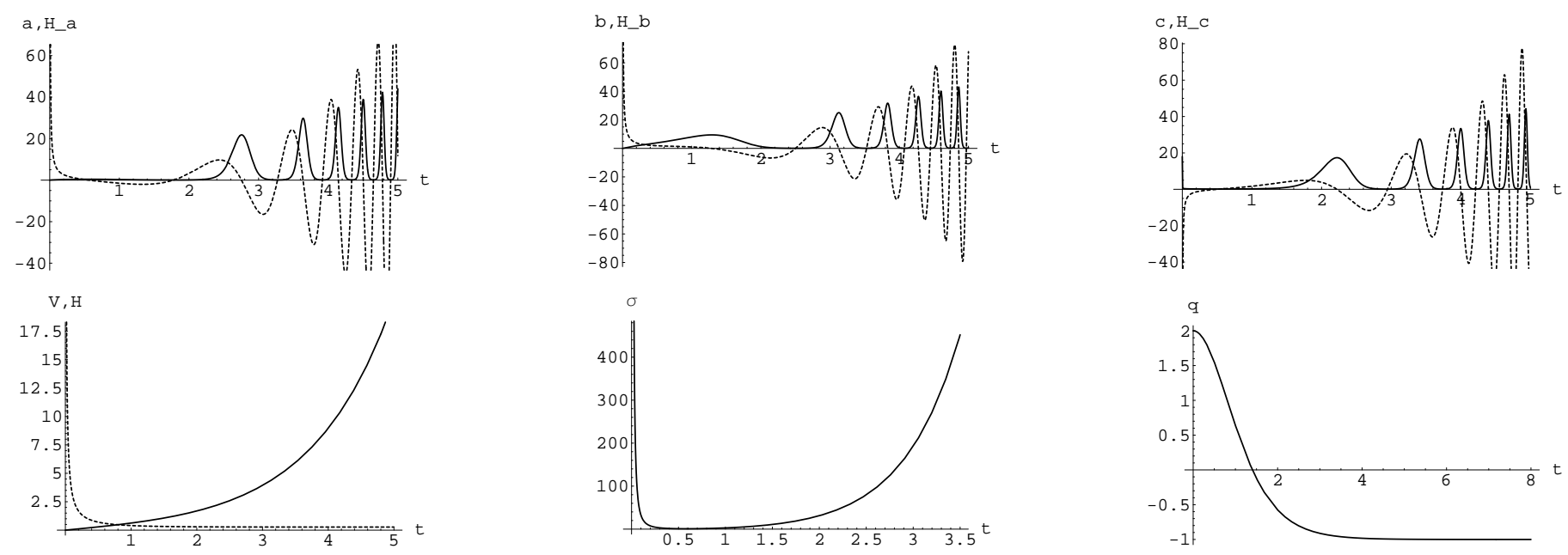

Figure 2: Top: Behavior of the scale factors $a(t), b(t)$ and $c(t)$ (solid lines) and their corresponding Hubble parameters (dashed lines) as a function of time. Bottom: The comoving volume $V(t)=e^{3 u}=a b c$ (solid line) and its corresponding Hubble parameter (dashed line), the shear scalar and the deceleration parameter. The figures are plotted for numerical values $\Lambda=1, p_{0 v}=1, p_{0 w}=2, \theta=0.25$ and $C_{1}=C_{2}=1$.

feature of this model is that its shear scalar which measures the degree of anisotropy in the space-time, behaves nonmonotonically. For small values of $t$, it decreases and tends to zero and then it increases monotonically, eventually diverging as $t \rightarrow \infty$. Therefore, the model under consideration does not satisfy the conditions for approaching isotropy. Since this behavior is not consistent with late time observations, it seems an additional mechanism is needed to modify this feature of the model. This is the subject of our forthcoming work and thus we do not deal with this issue here.

For deformed phase space of type II, using the (Poisson) commutation relations (45), the cosmological dynamics of the Bianchi-I model after applying the Hamiltonian constraint are

$$
\left\{\begin{array}{l}
\dot{u}=\{u, \mathcal{H}\}=-\frac{1}{12} \bar{\theta} e^{-3 u}\left(w p_{v}+v p_{w}\right)-\frac{1}{12} e^{-3 u} p_{u} \\
\dot{v}=\{v, \mathcal{H}\}=6 \Lambda \theta w e^{3 u}+\frac{1}{12} e^{-3 u} p_{v} \\
\dot{w}=\{w, \mathcal{H}\}=-6 \Lambda \theta v e^{3 u}+\frac{1}{12} e^{-3 u} p_{w} \\
\dot{p_{u}}=\left\{p_{u}, \mathcal{H}\right\}=-6 \Lambda e^{3 u} \\
\dot{p_{v}}=\left\{p_{v}, \mathcal{H}\right\}=6 \Lambda\left(\bar{\theta} w+\theta p_{w}\right) e^{3 u} \\
\dot{p_{w}}=6 \Lambda\left(\bar{\theta} v-\theta p_{v}\right) e^{3 u}
\end{array}\right.
$$

We see that this kind of deformed cosmology forms a system of nonlinear coupled differential equations which unfortunately cannot be solved analytically. In figure 3, employing numerical methods, we have shown the approximate behavior of the corresponding cosmological functions for typical values of the parameters and initial conditions respectively. From this figure it is seen that the cyclic behavior of the corresponding cosmology is almost like the case of type I deformation except that here the average deceleration parameter also behaves oscillatory which means that the Universe experiences a periodic sequence in which it decelerates and accelerates its expansion alternatively.

\section{Conclusions}

In this paper we have studied the possibility of emergence of a Bianchi type I cyclic cosmology in the framework of a deformed phase space of Lie-algebraic type. In the proposed model the phase space 

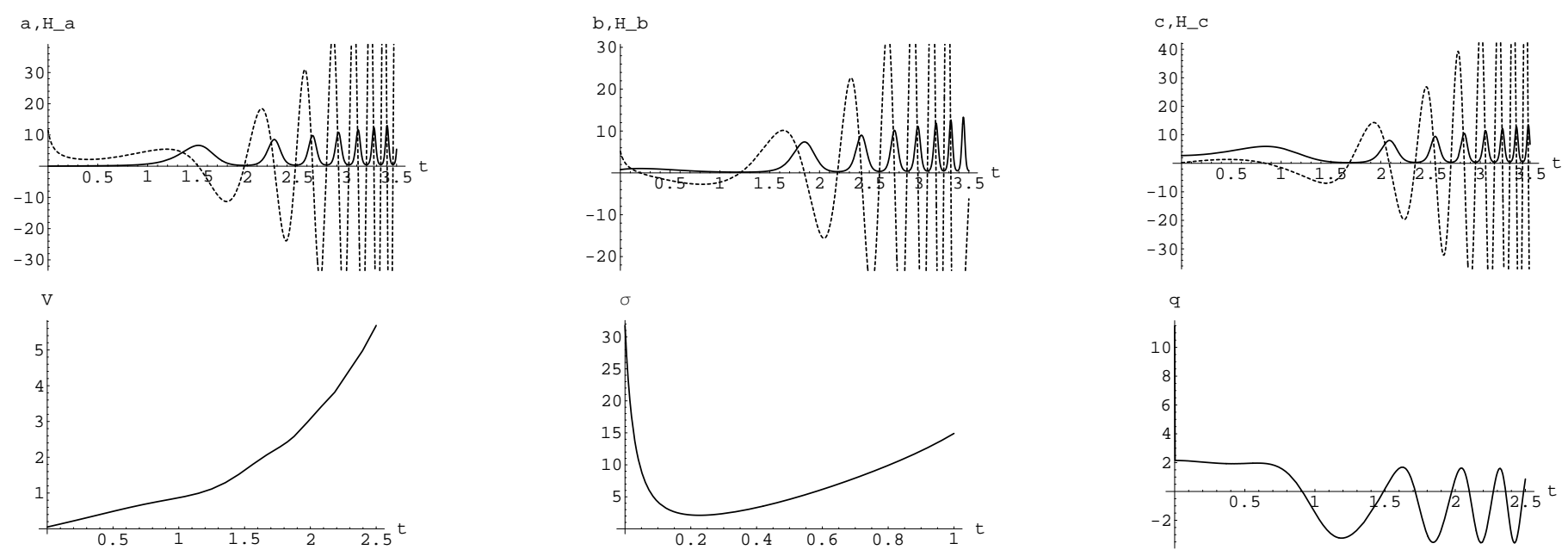

Figure 3: Up: Qualitative behavior of the scale factors $a(t), b(t)$ and $c(t)$ (solid lines) and their corresponding Hubble parameters (dashed lines) as a function of time. Bottom: The comoving volume $V(t)=e^{3 u}=a b c$, the shear scalar and the deceleration parameter. The figures are plotted for numerical values $\Lambda=1, \theta=0.5$ and $\bar{\theta}=0.5$. We take the initial conditions $u(0)=v(0)=w(0)=-1, p_{0 u}=-2, p_{0 v}=1.7$ and $p_{0 w}=1$. After examining other sets of initial conditions, we verify that this behavior repeats itself.

coordinates obey the (Poisson) commutation relations with a Lie-algebraic structure, the structure constants of which play the role of the deformation parameters. These constants are subject to conditions to guarantee the Jacobi identity which by solving them the noncommutative (deformation) parameters can be found. In this line we have followed Refs. [17] that offer two types of noncommutative parameters which satisfy the corresponding constraints. We investigated the Bianchi type I cosmology based on these two kinds of deformed phase space and compare the results with the conventional model. For the deformed model of the first kind we showed that while the behavior of the whole volume of the Universe and its deceleration parameter are the same as the consensus Bianchi I model, the evolution of its scale factors is quite different. In spite of the usual Bianchi I model in which the three scale factors increase monotonically, in the Lie-algebraically deformed model they evolve cyclically. This means that in the scenario proposed by the deformed model the scale factors of the Universe undergo an endless sequence of epochs which begin with a big-bang and end in a big-crunch, i.e., the size of the Universe in each direction bounces from contraction to reexpansion alternatively. We saw that this behavior causes a high degree of anisotropy as $t \rightarrow \infty$ in contrast to the late time observations and therefore it seems an additional mechanism is needed to modify this feature of the model. For the second kind of deformation parameters, since we could not solve the system of field equations analytically, we investigated the subject by employing numerical methods. Our numerical analysis in this case showed again an oscillatory behavior not only for the scale factors but also for the total deceleration parameter.

\section{Acknowledgements}

The authors are grateful to the anonymous referees for enlightening suggestions. B. V. would like to thank the research council of Azad University of Chalous for financial support.

\section{References}

[1] H. Snyder, Phys. Rev. 71 (1947) 38

[2] A. Connes, Noncommutative Geometry, (1994) (New York: Academic)

E. Witten, Nucl. Phys. B 460 (1996) 335

J.C. Varilly, An Introduction to Noncommutative Geometry, (1997) Lectures at EMS Summer 
School on NCG and Applications (Sept 2007) (arXiv: physics/9709045)

M.R. Douglas and N.A. Nekrasov, Rev. Mod. Phys. 73 (2001) 977 (arXiv: hep-th/0106048)

A. Konechny and A. Schwarz, Phys. Rep. 360 (2002) 353

B. Chakraborty, S. Gangopadhyay and A. Saha, Phys. Rev. D 70 (2004) 107707 (arXiv: hepth/0312292)

E. Harikumar, V.S. Kumar and A. Khare, Phys. Lett. B 589 (2004) 155 (arXiv: hep-th/0402064)

[3] S. Minwalla, M. Van Raamsdonk and N. Seiberg, J. High Energy Phys. JHEP02 (2000) 020 (arXiv: hep-th/9912072)

S.M. Carroll, J.A. Harvey, V.A. Kostelesky, C.D. Lane and T. Okamoto, Phys. Rev. Lett. 87 (2001) 141601 (arXiv: hep-th/0105082)

C.E. Carlson, C.D. Carone and R.F. Lebed, 2001 Phys. Lett. B 518 (2001) 201 (arXiv: hep$\mathrm{ph} / 0107291)$

C.E. Carlson, C.D. Carone and R.F. Lebed, Phys. Lett. B 549 (2002) 337 (arXiv: hep$\mathrm{ph} / 0209077)$

R.J. Szabo, Phys. Rep. 378 (2003) 207 (arXiv: hep-th/0109162)

M.R. Douglas and N.A. Nekrasov, Rev. Mod. Phys. 73 (2001) 977 (arXiv: hep-th/0106048)

[4] J. Gamboa, M. Loewe and J.S. Rojas, Phys. Rev. D 64 (2001) 067901 (arXiv: hep-th/0010220) A.E.F. Djemai and H. Smail, Commun. Theor. Phys. 41 (2004) 837 (arXiv: hep-th/0309006)

L. Mezincescu, Star operation in quantum mechanics Preprint (2000) hep-th/0007046

[5] J.M. Romero, J.A. Satiago and D. Vergara, Phys. Lett. A 310 (2003) 9 (arXiv: hep-th/0211165) M. Przanowski and J. Tosiek, Acta Phys. Pol. B 30 (1999) 179

A.E.F. Djemai, On noncommutative classical mechanics (2003) (arXiv: hep-th/0309034)

[6] J. Wess, in Noncommutative Structures in Mathematics and Physics, Proceedings of the NATO Advanced Research Workshop, Kiev, Ukraine September 24-28 (2000), Editors: S. Duplij and J. Wess (Springer 2001)

[7] B. Vakili, N. Khosravi and H.R. Sepangi, Class. Quantum Grav. 24 (2007) 931 (arXiv: grqc/0701075)

N. Khosravi, H.R. Sepangi and M.M. Sheikh-Jabbari, Phys. Lett. B 647 (2007) 219 (arXiv: hepth/0611236)

N. Khosravi, S. Jalalzadeh and H.R. Sepangi, Gen. Rel. Grav. 39 (2007) 899 (arXiv: grqc/0702067)

W. Guzman, M. Sabido and J. Socorro, On noncommutative minisuperspace and the Friedmann equations (arXiv: 0812.4251 [gr-qc])

H. Garcia-Compean, O. Obregon and C. Ramirez, Phys. Rev. Lett. 88 (2002) 161301 (arXiv: hep-th/0107250)

Y.F. Cai and Y.S. Piao, Phys. Lett. B 657 (2007) 1 (arXiv: gr-qc/0701114)

C. Bastos, O. Bertolami, N.C. Dias and J.N. Prata Phys. Rev. D 78 (2008) 023516 (arXiv: $0712.4122)$

M.V. Battisti and G. Montani, Phys. Lett. B 656 (2007) 96 (arXiv: gr-qc/0703025)

M.V. Battisti and G. Montani, Phys. Rev. D 77 (2008) 023518 (arXiv: 0707.2726)

B. Vakili and H.R. Sepangi, Phys. Lett. B 651 (2007) 79 (arXiv: 0706.0273 [gr-qc])

B. Vakili, Phys. Rev. D 77 (2008) 044023 (arXiv: 0801.2438 [gr-qc])

H.R. Sepangi, B. Shakerin and B. Vakili, Class. Quantum Grav. 26 (2009) 065003 (arXiv: 0901.3829 [gr-qc])

[8] R. Tolman, Relativity, Thermodynamics and Cosmology, Oxford, Clarendon (1934)

[9] P.J. Steinhart and N. Tutok, Phys. Rev. D 65 (2002) 126003 (arXiv: hep-th/0111098)

P.J. Steinhardt and N. Turok, : Nucl. Phys. Proc. Suppl. 124 (2003) 38 (arXiv: astro$\mathrm{ph} / 0204479)$ 
J. Khoury, P.J. Steinhardt and N. Turok, Phys. Rev. Lett. 92 (2004) 031302 (arXiv: hepth/0307132)

H.H. Xiong, Y.F. Cai, T. Qiu, Y.S. Piao and X. Zhang, Phys. Lett. B 666 (2008) 212 (arXiv: 0805.0413 [astro-ph])

H.H. Xiong, T. Qiu, Y.F. Cai, and X. Zhang, Mod. Phys. Lett. A 24 (2009) 1237 (arXiv: 0711.4469 [hep-th])

Y.F. Cai and E.N. Saridakis, Cyclic cosmology from Lagrange-multiplier modified gravity (arXiv: 1007.3204 [astro-ph])

[10] M. Novello and S.E. Bergliaffa, Phys. Rep. 463 (2008) 127 (arXiv: 0802.1634 [astro-ph])

[11] Ø. Grøn, Phys. Rev. D 32 (1985) 2522

Ø. Grøn and S. Hervik, Einstein's General Theory of Relativity, Springer, New York, (2007)

M.P. Ryan, Hamiltonian Cosmology (1972) (Berlin: Springer)

M.P. Ryan and L.C. Shepley, Homogeneous Relativistic Cosmologies (1975) (Princeton: Princeton University Press)

M. Henneaux, Phys. Rev. D 21 (1980) 857

[12] C.W. Misner, Phys. Rev. 186 (1969) 1319

[13] S.W. Hawking, Mon. Not. R. Asrton. Soc. 142 (1966) 129

M.P. Ryan, J. Math. Phys. 15 (1974) 812

G.E. Sneddon, J. Phys. A: Math. Gen. 9 (1976) 229

[14] C. Kiefer, Quantum Gravity (2007) (Oxford University Press)

[15] T. Christodoulakis, T. Gakis and G.O. Papadopoulos, Class. Quantum Grav. 19 (2002) 1013 (arXiv: gr-qc/ 0106065)

T. Christodoulakis and G.O. Papadopoulos, Quantum cosmology for the general Bianchi type II, VI (class A) and VII (class A) vacuum geometries (arXiv: gr-qc/0109058)

[16] D.C. Rodrigues, Phys. Rev. D 77 (2008) 023534 (arXiv: 0708.1168 [astro-ph])

S. Hervik, Class. Quantum Grav. 17 (2000) 2765 (arXiv: gr-qc/0003084)

B. Vakili and H.R. Sepangi, J. Cosmol. Astropart. Phys. JCAP0509 (2005) 008 (arXiv: grqc/0508090)

O. Akarsu and C.B. Kilinc, Some anisotropic Universes in the presence of imperfect fluid coupling with spatial curvature (arXiv: 1008.2543 [gr-qc])

B. Cropp and M. Visser, Any spacetime has a Bianchi type I spacetime as a limit, (arXiv: 1008.4639 [gr-qc])

M. Sharif and M.F. Shamir, Class. Quantum Grav. 26 (2009) 235020 (arXiv: 0910.5787 [gr-qc])

[17] Y.G. Miao, X.D. Wang and S.J. Yu, Classical Mechanics on Noncommutative Space with Liealgebraic Structure (arXiv: 0911.5227 [math-ph])

M. Daszkiewicz and C.J. Walczyk, Acta Phys. Polo. B 40 (2009) 293 (arXiv: 0812.1264 [hep-th])

M. Daszkiewicz and C.J. Walczyk, Phys. Rev. D 77 (2008) 105008 (arXiv: 0802.3575 [math-ph]) 